\title{
pN1 but not pN0/N2 predicts survival benefits of prophylactic cranial irradiation in small-cell lung cancer patients after surgery
}

\author{
Haitang Yang $^{1 \# \wedge}$, Mohammad Faisal Al-Hurani ${ }^{2 \#}$, Jianlin Xu ${ }^{3}$, Liwen Fan ${ }^{1}$, Ralph A. Schmid ${ }^{2}$ Heng Zhao ${ }^{1}$, \\ Feng Yao ${ }^{1}$
}

${ }^{1}$ Department of Thoracic Surgery, Shanghai Chest Hospital, Shanghai Jiao Tong University, Shanghai, China; ${ }^{2}$ Division of General Thoracic Surgery, Inselspital, Bern University Hospital, University of Bern, Bern, Switzerland; ${ }^{3}$ Department of Pulmonary, Shanghai Chest Hospital, Shanghai Jiao Tong University, Shanghai, China

Contributions: (I) Conception and design: H Yang, F Yao; (II) Administrative support: F Yao; (III) Provision of study materials or patients: MF AlHurani, J Xu, L Fan, H Zhao; (IV) Collection and assembly of data: MF Al-Hurani, J Xu; (V) Data analysis and interpretation: All authors; (VI) Manuscript writing: All authors; (VII) Final approval of manuscript: All authors.

\#These authors contributed equally to this work.

Correspondence to: Feng Yao, MD. Department of Thoracic Surgery, Shanghai Chest Hospital, Shanghai Jiao Tong University, West Huaihai 241, Xuhui District, Shanghai 200030, China. Email: feng.yao@shchest.org.

Background: Prophylactic cranial irradiation has been shown to reduce brain metastases and provide survival benefits in small-cell lung cancer (SCLC). However, its role in limited-stage SCLC patients after surgery remains unclear. Further, it is unknown whether the effect of prophylactic cranial irradiation is generalizable in these patients with different pathological nodal (N0-N2) stages, a state indicating the presence of tumor metastases.

Methods: We combined data from a single medical center and Surveillance, Epidemiology, and End Results database. Propensity score matching analyses were performed (1:2) to evaluate the role of prophylactic cranial irradiation in SCLC patients after surgery. Cox proportional hazards regression model was used to identify predictors of survival.

Results: 124 (18.7\%) out of 664 surgically-treated SCLC patients received prophylactic cranial irradiation treatment. Within the entire cohort, multivariate Cox regression analysis identified dataset source, age, pathological $\mathrm{T}$ and $\mathrm{N}$ stages, adjuvant chemotherapy, resection type, and histology as independent prognostic factors for overall survival. Prophylactic cranial irradiation appeared to be associated with a better overall survival, but the difference is marginally significant $(\mathrm{P}=0.063)$. Further, we stratified patients based on the pathological N0-N2 stages using propensity score matching analyses, which showed that prophylactic cranial irradiation treatment was superior to non-prophylactic cranial irradiation treatment for surgicallytreated SCLC patients with N1 stage only (univariate analysis: $\mathrm{P}=0.026$; multivariate $\mathrm{Cox}$ : $\mathrm{P}=0.004$ ), but not N0/N2 stage (univariate analysis: $\mathrm{P}=0.65$ and $\mathrm{P}=0.28$, respectively; multivariate Cox: $\mathrm{P}=0.99$ and $\mathrm{P}=0.35$, respectively).

Conclusions: Prophylactic cranial irradiation provides survival benefits for SCLC patients with pN1 after surgery but not with pathological N0/N2 stage. Our findings may provide helpful stratifications for clinical decision-making of prophylactic cranial irradiation intervention in SCLC patients.

Keywords: Small-cell lung cancer (SCLC); surgery; nodal metastasis; prophylactic cranial irradiation

Submitted Oct 18, 2020. Accepted for publication Jan 12, 2021.

doi: 10.21037/atm-20-6984

View this article at: http://dx.doi.org/10.21037/atm-20-6984

\footnotetext{
^ ORCID: 0000-0002-8732-5910.
} 


\section{Introduction}

Small-cell lung cancer (SCLC) is a highly aggressive neuroendocrine tumor, characterized by a dismal prognosis, rapid growth, and early distant metastasis $(1,2)$. One of the distinguishing features of SCLC is the tendency to metastasize to the brain (3). Approximately $10 \%$ of SCLC patients have brain metastases (BM) at diagnosis, and this percentage expands over time, reaching more than $50 \%$ in 2 -year survivors (3). However, only $27 \%$ of SCLC patients with BM respond to systemic chemotherapy. This resistance is mainly attributed to the presence of the blood-brain barrier $(4,5)$.

Given the high frequency of BM and propensity for micrometastasis with early-stage disease, prophylactic cranial irradiation (PCI) has been shown to decrease the development of BM and prolong survival in SCLC patients $(6,7)$. PCI has ever been recommended as an element of standard care for extensive- (ES-SCLC) or limitedstage SCLC (LS-SCLC) patients with complete/partial remission after first-line treatment (8). However, a recent randomized clinical trial challenged the role of PCI in ESSCLC patients with any response to initial chemotherapy, in that PCI significantly reduces the incidence of BM ( $48 \%$ vs. $69 \% ; \mathrm{P}<0.0001)$, but does not improve overall survival $(\mathrm{P}=0.094)$, compared with observation group (9). Further, recent evidence showed no significant survival benefit from PCI in ES-/LS-SCLC patients without BM confirmed by brain magnetic resonance imaging (MRI) after initial treatment (9-11). Thus, how to stratify SCLC patients for precise $\mathrm{PCI}$ interventions remains a question.

Additionally, increasing evidence suggests a critical role of surgery in patients with resectable LS-SCLC tumors, along with adjuvant chemotherapy, significantly prolong survival $(3,12-15)$. Accordingly, the National Comprehensive Cancer Network guideline has recommended surgery for LS-SCLC patients at an early-stage without lymph node metastasis (T1-2, N0). Besides, some recent studies have demonstrated that PCI can decrease the incidence of $\mathrm{BM}$ and improve the prognosis of patients with resectable LS-SCLC after surgery $(14,16-18)$; however, the target populations that can considerably benefit from PCI is not yet specified. Of note, PCI is known to be related to neurotoxicity, which needs to be critically considered before clinical employment. Since there is little information on PCI after resection of early SCLC (19), whether to perform PCI for all SCLC patients after surgery is of high importance that needs further research. Additionally, few studies have investigated the relationship between PCI treatment effect and different pathological nodal (pN0-N2) status at surgery, a critical factor indicating the occurrence of tumor metastasis and influencing the prognosis of cancer patients.

In this study, we sought to determine the effect of PCI on LS-SCLC patients after surgery, and which subsets, according to pN0-N2 status, can benefit from PCI. For this purpose, we combined data from a single medical center and Surveillance, Epidemiology, and End Results (SEER) program, given the sparsity of surgically-resected LS-SCLC cases and a generally low incidence of PCI interventions for this population.

We present the following article in accordance with the STROBE reporting checklist (available at http://dx.doi. org/10.21037/atm-20-6984).

\section{Methods}

\section{Patient selection}

This study was approved by the Institutional Review Board of Shanghai Chest Hospital (SCH) (Shanghai, China). Clinical data of SCLC (between 2006 and 2014), including age at diagnosis, sex, type of resection (sublobar resection/ lobectomy), histology subtype [pure/combined SCLC (combined non-SCLC components)], tumor size, the extent of lymph node involvement, adjuvant thoracic radiotherapy and chemotherapy. Tumors were staged based on the eighth tumor node metastasis classification. To reduce the number of prognosis-influencing factors, we targeted the group of surgically-treated patients with complete resections. The inclusion criteria were as follows: (I) pathologicallyconfirmed SCLC; (II) surgery with complete resection as initial treatment for primary SCLC tumor. The exclusion criteria were: (I) patients who had positive surgical margins (including microscopically-positive and macroscopicallypositive); (II) patients without survival and therapy (particularly PCI treatment) details; (III) patients with N3/stage IV disease; (IV) postoperative survival time less than three months; (V) SCLC was not identified as the first primary cancer (to exclude the cases whose survival might be significantly affected by the prior cancer). Overall survival (OS) was defined as the time from diagnosis until death from any cause or last follow-up visit. The study was conducted in accordance with the Declaration of Helsinki (as revised in 2013). The participants gave informed consent before taking part. The study was approved by ethics board of Shanghai Chest Hospital (FS202007). 


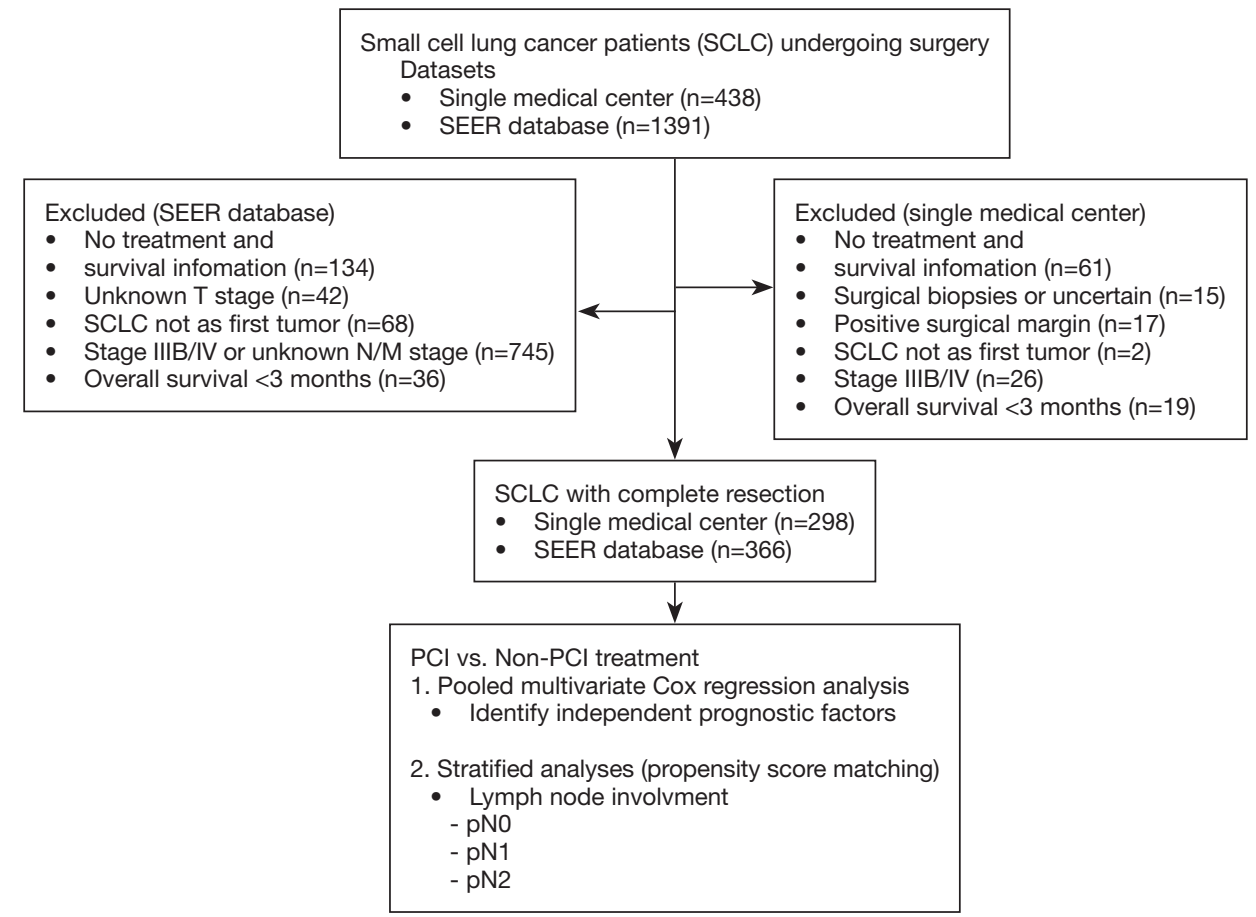

Figure 1 The workflow of this study.

In this study, we used the SEER public database from 1975-2016 datasets (November 2018 submission) for this study. We requested the data from the public SEER database portal (www.seer.cancer.gov), then we received the approval to get access to the database. Histology and site of the disease are coded in SEER according to the International Classification of Diseases (ICD) for Oncology, Edition 3 (ICD-O-3). Data of patients with SCLC (ICD0-3 histology code 804.1) of the lung (ICD-0-3 site code c34.0-c34.9) were extracted. Then the data of patients undergoing complete resection were filtered and analyzed. We included cases only diagnosed between 1988 and 1997 because the delivery of whole-brain radiotherapy was not specifically recorded during other years $(20,21)$. Furthermore, we excluded SCLC patients with N3/stage IV or unknown $\mathrm{T} / \mathrm{N}$ stage disease, those not diagnosed with SCLC as the first primary cancer, or survived less than three months postoperatively.

A total of 664 SCLC patients, including 298 from SCH and 366 from the SEER database, were included for analysis.

\section{Study design}

Initially, we investigated the effect of PCI treatment based on the entire patient cohort, then further analysis according to the pN0-N2 status of surgically-resected SCLC tumors was performed. The workflow was summarized in Figure 1.

\section{Statistical analysis}

Normally distributed continuous variables were presented as the mean $\pm \mathrm{SD}$, otherwise presented as the median and range. Categorical variables were shown as numbers and percentages. Fisher's exact test and a chi-square test were used to compare categorical variables, while a t-test was used to compare continuous variables. Survival curves were estimated using the Kaplan-Meier method and compared using the log-rank test. The propensity score matching (PSM) analyses (1:1 or 1:2; by nearest-neighbor matching) was performed to control the differences in baseline characteristics between two groups (PCI vs. nonPCI treatment) (22), such as the source of datasets (SHC vs. SEER), age, sex, histology subtype, type of resection, adjuvant thoracic radiotherapy and chemotherapy, and pathological characteristics. In the case of good matching (1:1 and 1:2), 1:2 matching was preferred to achieve sufficient statistical power, given the small sample size in our study. Then, univariate Kaplan-Meier and multivariate 
Table 1 Clinical characteristics of the entire cohort

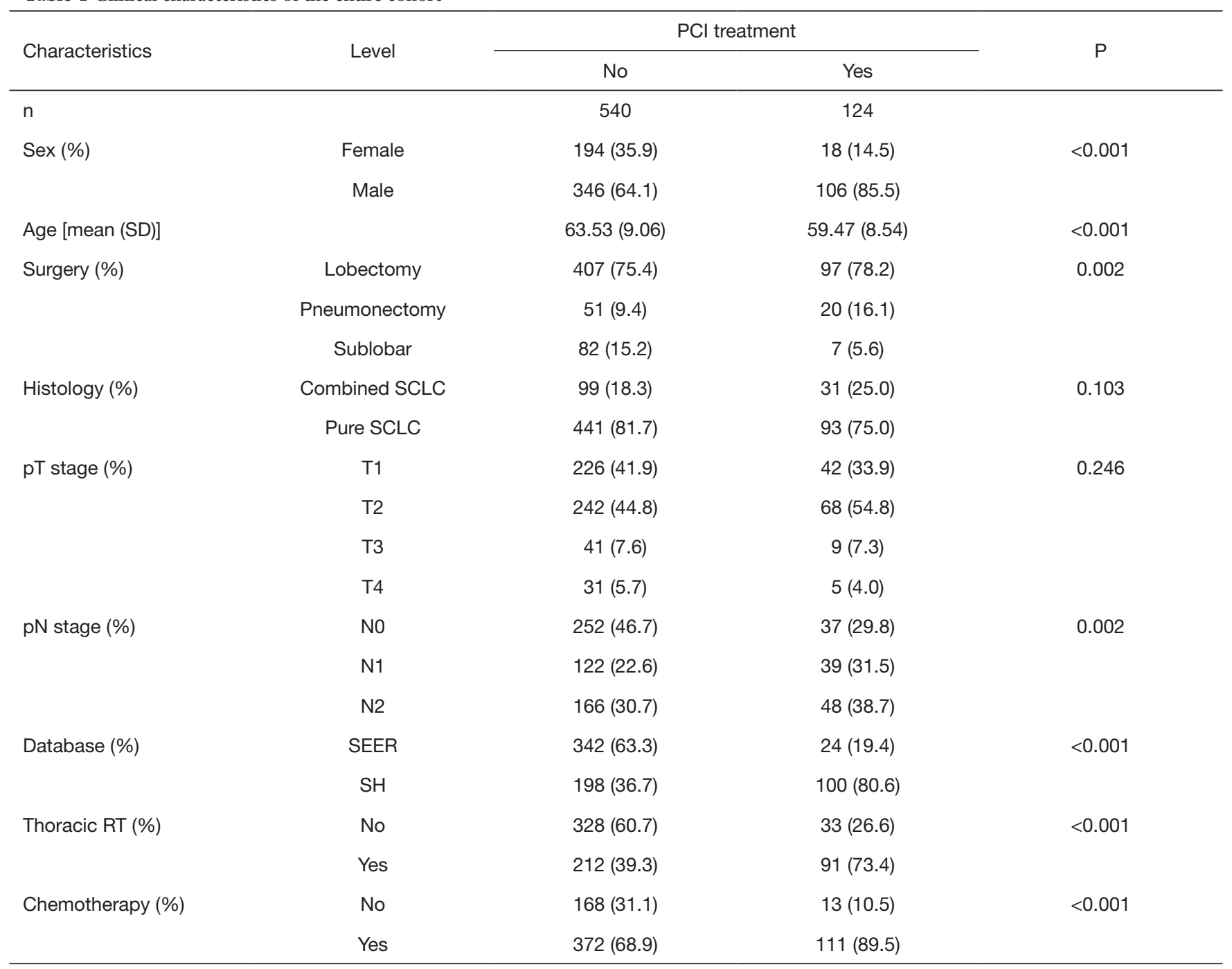

pT, pathological T; pN, pathological N; RT, radiotherapy; SHC, Shanghai Chest Hospital; Combined SCLC means the presence of non-SCLC components (>10\%) within tumor.

Cox regression analyses were performed on the subgroup of matched patients. All tests were two-sided, and a $\mathrm{P}$ value $<0.05$ was considered to indicate statistical significance. Statistical analyses were performed using $\mathrm{R}$ software (version 3.6.3). PSM and survival analyses were performed using $\mathrm{R}$ packages 'MatchIt' (for PSM), 'survival' and 'survminer' (for survival), respectively.

\section{Results}

\section{Patient characteristics and prognostic factors}

The clinical characteristics of all SCLC patients included in this study were shown in Table 1. Of the 664 surgicallytreated SCLC patients included, 124 (18.7\%) received PCI. Multivariate Cox regression analysis (Figure $2 A$ ) identified SHC-based dataset [hazards ratio $(\mathrm{HR})=1.35$, $95 \%$ confidence interval (CI): 1.04-1.75], age (61-70 years, $\mathrm{HR}=1.46,95 \%$ CI: $1.03-2.06 ; 71-80$ years: $\mathrm{HR}=2.19$, 95\% CI: $1.51-3.18)$, high pT (T2: HR $=1.25,95 \%$ CI: 1.03-1.53; T3: HR =2.13, 95\% CI: 1.49-3.05; T4: HR $=3.65,95 \%$ CI: $2.46-5.41)$, high $\mathrm{pN}(\mathrm{pN} 1: \mathrm{HR}=1.98$, 95\% CI: $1.55-2.52$; pN2: HR $=2.36,95 \%$ CI: $1.86-2.99$ ), adjuvant chemotherapy (HR $=0.62,95 \%$ CI: 0.51-0.77), type of resection (sublobar resection: $\mathrm{HR}=1.36,95 \% \mathrm{CI}$ : $1.05-1.77$ ), and histology (pure SCLC: $\mathrm{HR}=1.40,95 \% \mathrm{CI}$ : 
A

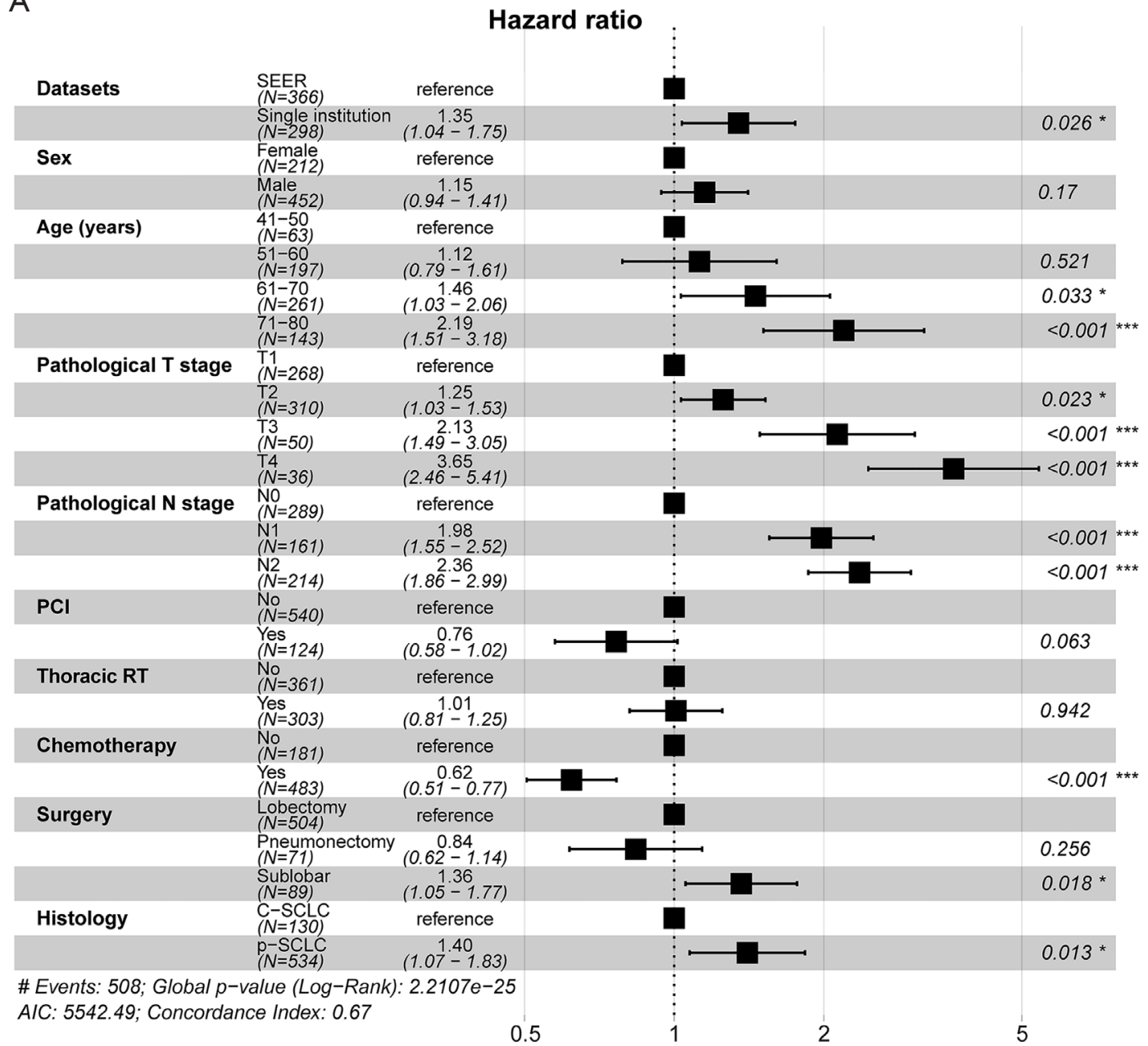

B
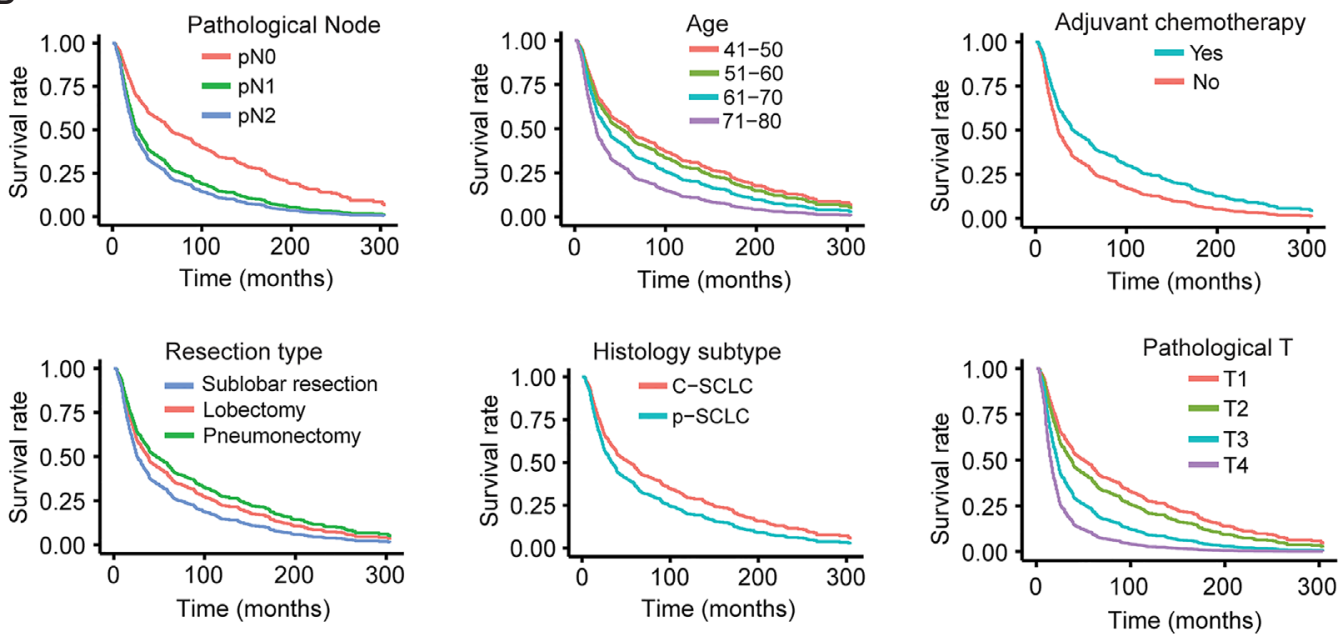

Figure 2 Prognostic factors of small-cell lung cancer (SCLC) patients after surgery. (A) Forest plot shows the independently prognosisinfluencing factors identified by multivariate Cox regression analysis; (B) Covariates-adjusted Kaplan-Meier analyses of the indicated factors in the entire SCLC patient cohort. 
1.07-1.83) as independent factors for OS. PCI appeared to be associated with a better OS, although the difference was not significant (HR $=0.76,95 \% \mathrm{CI}: 0.58-1.02, \mathrm{P}=0.063$ ). Covariates-adjusted Kaplan-Meier analyses of these prognosis-influencing factors were shown in Figure $2 B$.

\section{The association between PCI effect and pNO-N2 stage}

Tumor-positive lymph nodes are closely associated with distant metastasis and poor prognosis. Next, we sought to investigate whether the extent of lymph node involvement relates to the PCI effect. Thus, we performed PSM analyses $(1: 2)$ to evaluate the role of PCI in SCLC patients based on pN0-N2 stratifications.

\section{pNo}

After matching the baseline characteristics (Figure 3A), PSM analyses showed that there was no significant survival difference $(\mathrm{P}=0.65)$ between $\mathrm{PCI}$ and non-PCI group at the pN0 stage (Figure 3B). Further, multivariate Cox regression analysis identified age $(71-80$ years: $\mathrm{HR}=4.46,95 \%$ CI: 1.45-13.7) and pT (T3: HR =3.20, 95\% CI: 1.30-7.9), but not PCI (HR $=1.00,95 \%$ CI: $0.57-1.7)$, as significantly independent factors for OS (Figure 3C). The co-variatesadjusted survival curve of $\mathrm{pN} 0$-stage SCLC patients with and without PCI treatment was shown in Figure S1A.

\section{pN1}

Concerning patients with the pN1 stage, PSM analysis (Figure $4 A$ ) showed that the PCI group had significantly better OS than the non-PCI group $(\mathrm{P}=0.026$; Figure $4 B)$. Also, multivariate Cox regression analysis identified age (51-60 years: HR $=2.40,95 \%$ CI: $1.03-5.61)$ and pT (T4: $\mathrm{HR}=4.52$, 95\% CI: $1.54-13.21$ ), as well PCI as (HR $=0.43$, 95\% CI: 0.24-0.76), as significantly independent factors for OS (Figure 4C). The co-variates-adjusted survival curve of pN1-stage SCLC patients with and without PCI treatment was shown in Figure S1B.

\section{pN2}

Within the pN2-stage, we did not observe the superiority of PCI treatment after performing PSM (Figure $5 A$ ), as there was no survival advantage in the PCI group $(\mathrm{P}=0.28$; Figure $5 B)$. Additionally, multivariate Cox regression analysis only identified pT (T2: HR =2.80, 95\% CI: $1.542-5.08$; T3: HR $=4.82$, 95\% CI: $1.950-11.93$; T4: HR $=5.83$, 95\% CI: $1.783-15.76)$ and adjuvant chemotherapy ( $\mathrm{HR}=0.16,95 \%$
CI: $0.073-0.33$ ), rather than PCI (HR $=0.80,95 \%$ CI: $0.498-1.28)$, as significantly independent factors for OS (Figure 5C). The co-variates-adjusted survival curve of pN2stage SCLC patients with and without PCI treatment was shown in Figure S1C.

Additionally, given that the above evidence showed $\mathrm{pN} 1$ as a potential biomarker predicting the survival benefit from the PCI treatment, we then separately analyzed the two cohorts by focusing on the $\mathrm{pN} 1$ stage. Cox multivariate analysis demonstrated that $\mathrm{pN} 1$ stage significantly predicts the OS of resected SCLC from our single institutional dataset (Figure S2A) but not SEER (Figure S2B). The difference can be explained by the fact that only a small proportion of resected SCLC cases $(n=5)$ undergoing PCI treatment in the SEER database. In contrast, in our institutional dataset, 34 of 78 resected SCLC cases received PCI treatment after surgery.

\section{Discussion}

SCLC is a highly aggressive malignancy with a tendency for early dissemination (1). Despite the initial sensitivity to chemo-radiotherapy, treatment usually fails because of BM (4). The keystone in treating LS-SCLC patients depends on the use of chemotherapy (etoposide and cisplatin) and chest radiotherapy. This regimen is followed by PCI when the patient has good performance status (8). The importance of PCI in controlling BM was previously recognized $(6,7)$, but challenged recently (9), leading to its controversy.

\section{The role of PCI in early-stage SCLC after surgery}

Recently, increasing evidence has shown the critical role of surgery in SCLC patients, even at an advanced p-IIIA-N2 stage $(12,14,15,23)$. Due to the paucity of cases undergoing surgery, the effect of PCI on SCLC patients who underwent surgical resection for the primary tumor remains unclear. We and other groups have shown that PCI could improve OS and decrease BM in SCLC patients with p-II/III stage, but not p-I stage, which is likely due to the low incidence of $\mathrm{BM}$ in patients with p-I stage (16-18,24). Specifically, recent data from our center showed that of the 146 surgically resected SCLC patients without lymph node involvement (pN0), only $11.6 \%(17 / 146)$ of patients developed BM, and PCI does not reduce the risk of cerebral recurrence of resected pT1-2N0M0 SCLC (17). These data suggest that PCI may play a limited role in the very early-stage SCLC. 
A

SCLC with NO stage Distribution of Propensity Scores

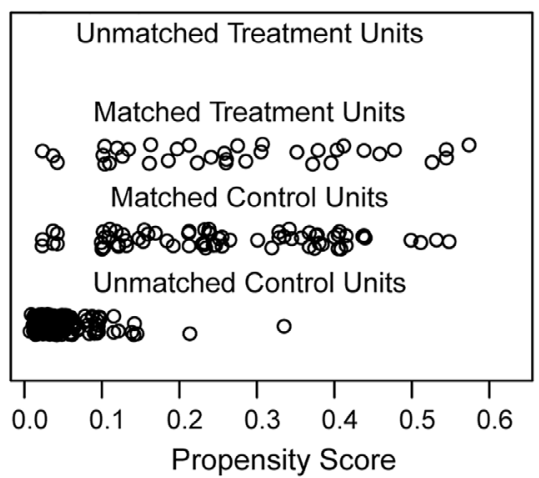

B

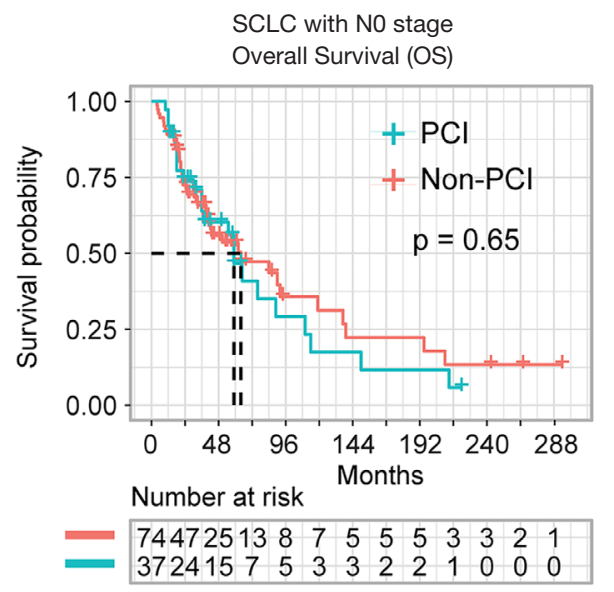

C

\section{Hazard ratio}

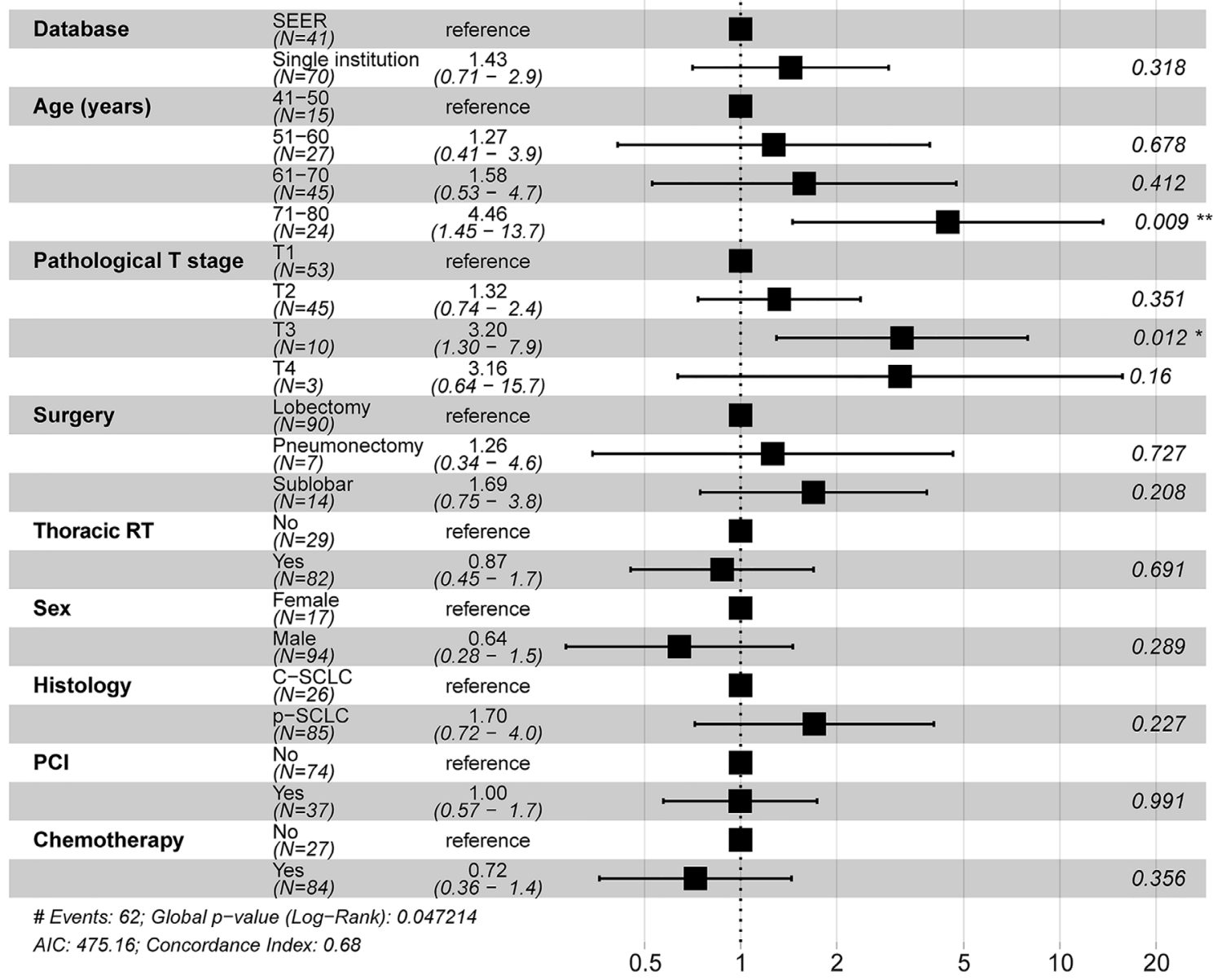

Figure 3 PCI treatment for $\mathrm{pN} 0$ stage small-cell lung cancer (SCLC) patients after surgery. (A) Distribution of propensity matching scores (PSM) between pN0 stage SCLC patients with and without PCI (prophylactic cranial irradiation) treatment; (B) PSM-based Kaplan-Meier analyses of SCLC patients with and without PCI; (C) Forest plot shows the independently prognosis-influencing factors identified by multivariate Cox regression analysis in PSM-matched (1:2) pN0 SCLC patients. 
A

SCLC with pN1 Distribution of Propensity Scores

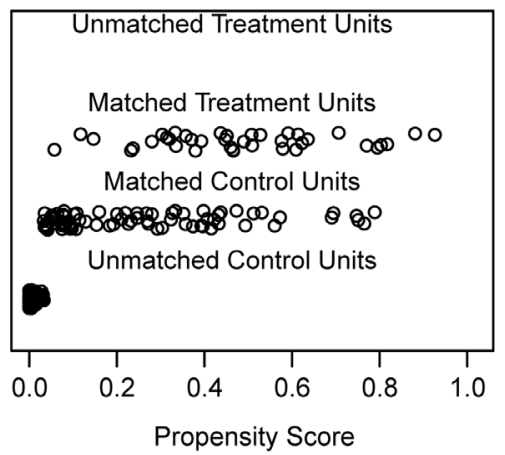

B

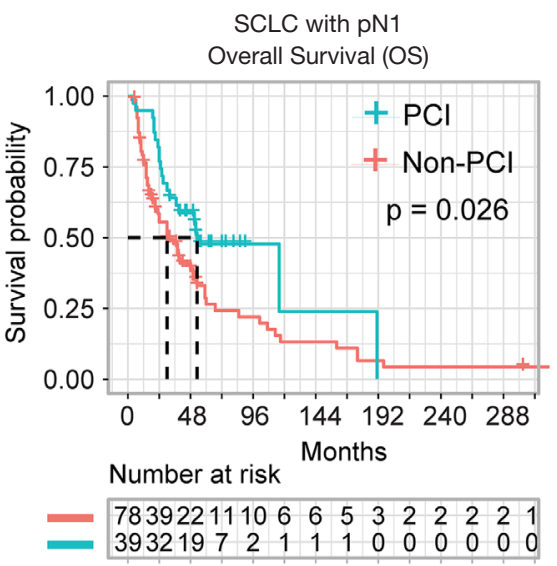

C

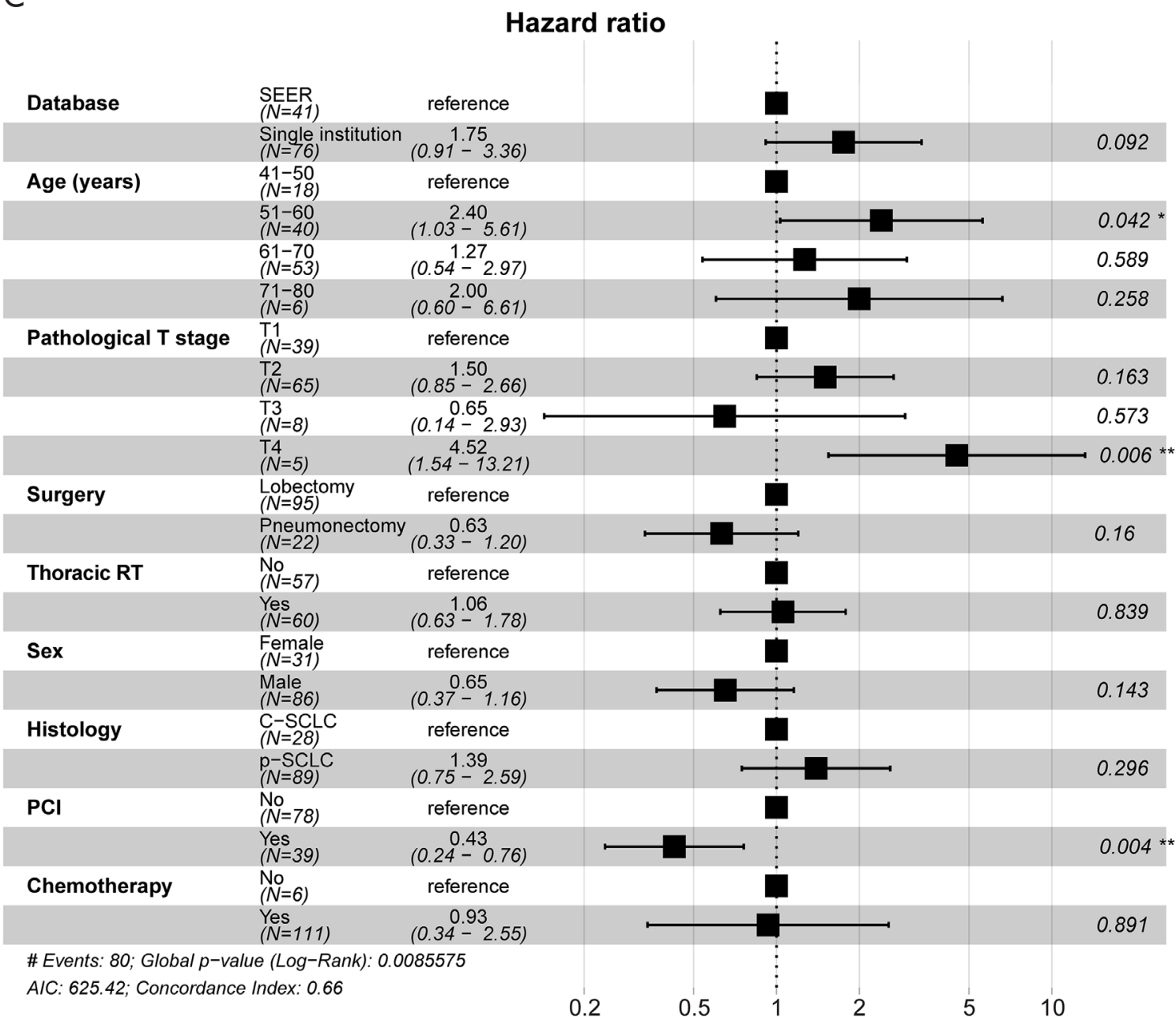

Figure 4 PCI treatment for pN1 stage small-cell lung cancer (SCLC) patients after surgery. (A) Distribution of propensity matching scores (PSM) between pN1 stage SCLC patients with and without PCI (prophylactic cranial irradiation) treatment; (B) PSM-based Kaplan-Meier analyses of SCLC patients with and without PCI; (C) Forest plot shows the independently prognosis-influencing factors identified by multivariate Cox regression analysis in PSM-matched (1:2) pN1 SCLC patients. 
A

SCLC with pN2

Distribution of Propensity Scores

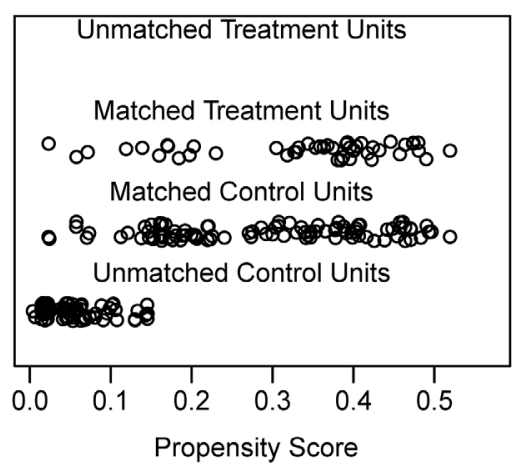

B

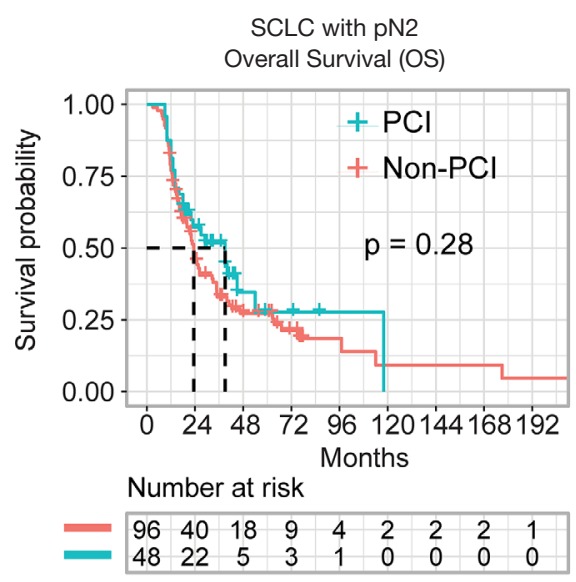

C

Hazard ratio

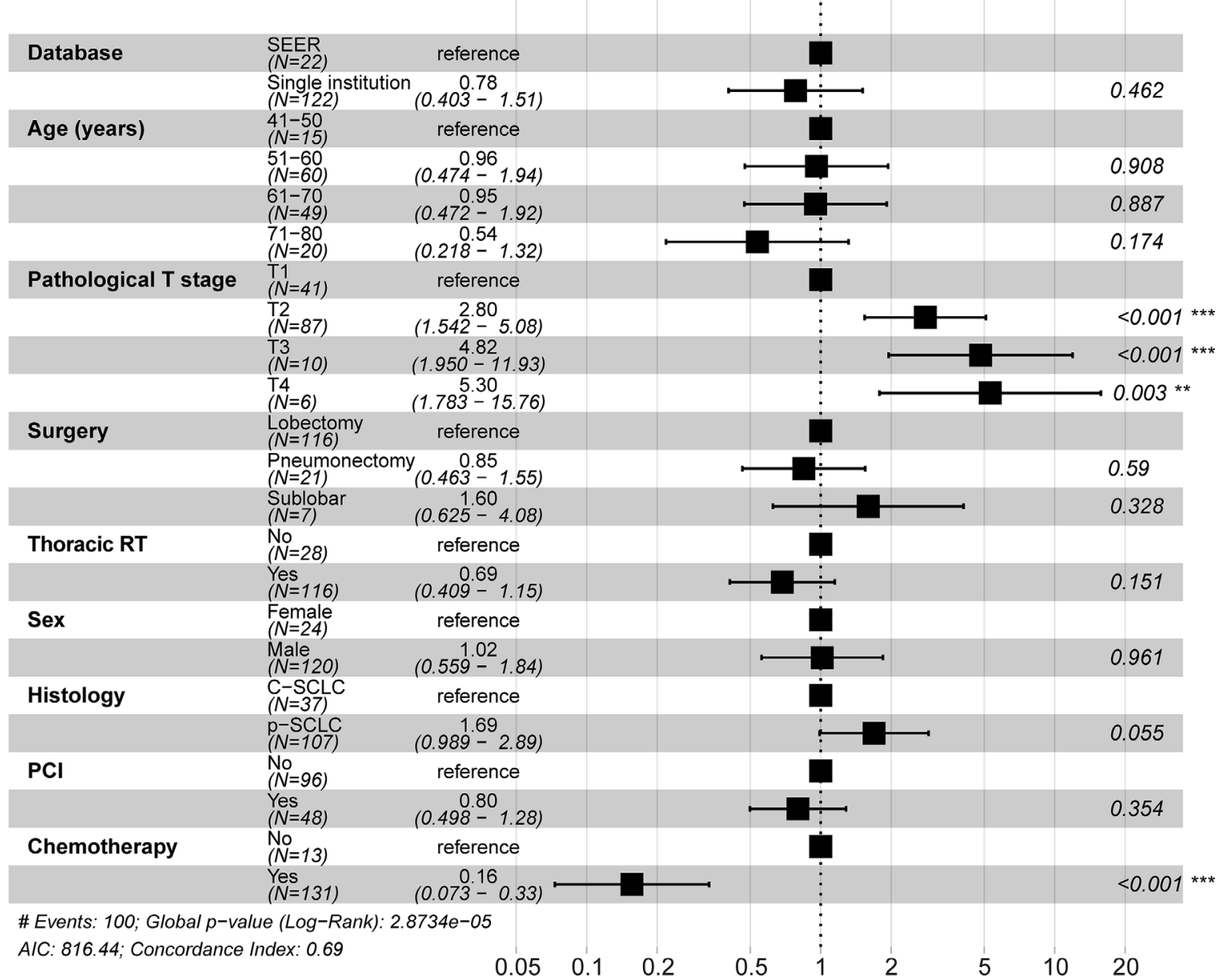

Figure 5 PCI treatment for pN2 stage small-cell lung cancer (SCLC) patients after surgery. (A) Distribution of propensity matching scores (PSM) between pN2 stage SCLC patients with and without PCI (prophylactic cranial irradiation) treatment; (B) PSM-based Kaplan-Meier analyses of SCLC patients with and without PCI; (C) Forest plot shows the independently prognosis-influencing factors identified by multivariate Cox regression analysis in PSM-matched (1:2) pN2 SCLC patients. 


\section{The role of PCI in advanced-stage SCLC with lymph node metastasis after surgery}

Identifying subsets of SCLC patients who can benefit from PCI intervention is an urgent need and remains a clinical challenge. Here, we investigated the role of PCI in surgically-treated SCLC, stratifying patients based on the extent of lymph node involvement ( $\mathrm{pN} 0-\mathrm{N} 2)$, given that lymph node involvement is tightly related to tumor metastasis. The stratifications were biologically and clinically relevant, and could also reduce the dimension of confounding factors. Supporting this notion, a recent study by Resio et al. analyzed data of 859 SCLC patients who underwent complete resection as the primary treatment (18). Subset analyses showed that a survival benefit from PCI was evident only in patients with positive lymph node involvement (18). However, it is unclear whether patients with different extent of lymph node involvement (e.g., $\mathrm{pN} 1 / \mathrm{N} 2)$ have differential survival benefits after PCI treatment (18). In our study, we found that PCI treatment could provide a survival advantage in patients at $\mathrm{pN} 1$ but not $\mathrm{pN} 0 / \mathrm{N} 2$ stage. This survival benefit was evident from PSM-based univariate and multivariate Cox analyses. In general, pN0 belongs to the p-I stage that associates with a low incidence of BM (16-18,24), which could explain the unbeneficial role of PCI treatment in this subgroup. Additionally, we observed that PCI was not superior to the non-PCI group in patients with $\mathrm{pN} 2$, which predominantly belongs to p-stage III. This observation appeared to be at odds with previous studies demonstrating survival benefits from PCI treatment in p-stage II-III SCLC patients $(16,18)$, which might be due to the higher heterogeneity of patients within $\mathrm{p}-\mathrm{III}$ classification than that of $\mathrm{pN} 2$. Also, previous study cohorts were not well-matched between the PCI and non-PCI treatment group, given that PSM analyses used in this study could reduce the bias by confounding factors (25). Further, patients with a more advanced pN2-stage might die of multiple dysfunctions of other metastasized organs other than the BM alone, in that SCLC tumors at distinct stages have varying metastatic patterns, leading to different prognosis $(26,27)$. Milovanovic et al. demonstrated that SCLC metastases were most commonly observed in the liver (more than 35\%), followed by adrenal glands (20\%), bone (17.6\%), brain (13.7\%), and myocardium (13.7\%) (27). Of note, evidence from the same study showed that in SCLC patients who survived less than one year after diagnosis, liver metastases were the most common (33\%), followed by intestinal metastases (22\%). Further studies are required to investigate whether the extent of lymph node involvement predicts different patterns of distant metastasis and organ-specific death in SCLC patients after surgery.

Notably, many research groups, including ours, showed some inconsistencies between clinical and pathological stages $(12,28,29)$, which might be due to that SCLC is highly aggressive with a high propensity for occult metastasis. Final histopathological analysis upstaged about $20 \%$ of SCLC patients with c-stage I/II to p-III $(12,29)$, thus, indicating that $\mathrm{p}$ - rather than c-stage is a more reliable marker to guide PCI treatment based on our findings.

\section{Neurotoxicity following PCI in SCLC}

However, the potential advantages of PCI should be balanced with possible side-effects. One of the side-effects related to this treatment modality is neurotoxicity. Briefly, all patients have the chance to develop acute toxic effects of PCI, like alopecia, headache, ear infection, fatigue, and nausea (30). Previous trials found that PCI is associated with a decrease in self-reported cognitive functioning and (Hopkins verbal learning test)-Recall and delayed recall at 6 and 12 months, especially in those above ages of 60 (4). Further, the optimal dose of PCI for LS-SCLC patients needs to be considered, as a higher dose of PCI is associated with more neurotoxic effects. A previous clinical trial showed that compared to the standard-dose PCI in patients with LS-SCLC, a higher-dose PCI is not associated with a significant reduction in the total incidence of brain metastases, but with a considerable increase in mortality (31). Thus, before applying PCI, we should weigh the balance between the benefits and risks of this treatment modality.

Therefore, it is critical to determine the subgroups that will not benefit from the treatment, thereby avoiding PCItreatment-related neurocognitive impairment. Although the neurotoxic data of SCLC patients after PCI treatment in the current study were not available, we identified that only a subgroup of SCLC patients ( $\mathrm{p}-\mathrm{N} 1$ stage) after complete resection could benefit from PCI treatment, which might provide new insights into SCLC patient selection for PCI treatment.

\section{Limitations}

The major limitation of this study is the retrospective nature. Particularly, the combined datasets are from different periods, which, however, might not be critical, given that the treatment strategies for SCLC have not 
changed significantly in the past three decades, and that we incorporated dataset source as a confounding factor for adjustment in the multivariate Cox and PSM analyses. Furthermore, some potentially prognosis-influencing factors, such as smoking history (in SHC dataset, 90\% of SCLC patients have smoking history), the performance status of patients, and the regimens and cycles of chemotherapy, are lacking in the SEER databases. Besides, baseline MRI scans are not available, as recent studies demonstrated no significant survival benefit from PCI in SCLC patients without BM confirmed by brain MRI after initial treatment (9-11).

\section{Conclusions}

In summary, our study showed PCI provided survival benefits for stage N1 but not N0/N2 SCLC patients after surgery. The results of this study suggest that the use of the extent of lymph node involvement may be helpful for clinical decision-making of PCI intervention in SCLC patients after surgery. More studies are needed not only to investigate the effect of PCI treatment in SCLC patients after complete resection but also whether the extent of lymph node involvement predicts different patterns of metastasis. Since only a minority of SCLC patients undergo surgery, a well-designed, multi-center, prospective study is required.

\section{Acknowledgments}

The authors acknowledge the efforts of the Surveillance, Epidemiology, and End Results (SEER) Program database. Funding: This research was supported by the Science and Technology Development project of Shanghai Chest Hospital (to Y Feng).

\section{Footnote}

Reporting Checklist: The authors have completed the STROBE reporting checklist. Available at http://dx.doi. org/10.21037/atm-20-6984

Data Sharing Statement: Available at http://dx.doi. org/10.21037/atm-20-6984

Conflicts of Interest: All authors have completed the ICMJE uniform disclosure form (available at http://dx.doi. org/10.21037/atm-20-6984). The authors have no conflicts of interest to declare.

Ethical Statement: The authors are accountable for all aspects of the work in ensuring that questions related to the accuracy or integrity of any part of the work are appropriately investigated and resolved. The study was conducted in accordance with the Declaration of Helsinki (as revised in 2013). The participants gave informed consent before taking part. The study was approved by ethics board of Shanghai Chest Hospital (FS202007).

Open Access Statement: This is an Open Access article distributed in accordance with the Creative Commons Attribution-NonCommercial-NoDerivs 4.0 International License (CC BY-NC-ND 4.0), which permits the noncommercial replication and distribution of the article with the strict proviso that no changes or edits are made and the original work is properly cited (including links to both the formal publication through the relevant DOI and the license). See: https://creativecommons.org/licenses/by-nc-nd/4.0/.

\section{References}

1. Gazdar AF, Bunn PA, Minna JD. Small-cell lung cancer: what we know, what we need to know and the path forward. Nat Rev Cancer 2017;17:725-37.

2. Hamilton G, Rath B, Ulsperger E. A review of the role of surgery for small cell lung cancer and the potential prognostic value of enumeration of circulating tumor cells. Eur J Surg Oncol 2016;42:1296-302.

3. Maeng CH, Song JU, Shim SR, et al. The Role of Prophylactic Cranial Irradiation in Patients With Extensive Stage Small Cell Lung Cancer: A Systematic Review and Meta-Analysis. J Thorac Oncol 2018;13:840-8.

4. Nakahara Y, Sasaki J, Fukui T, et al. The role of prophylactic cranial irradiation for patients with small-cell lung cancer. Jpn J Clin Oncol 2018;48:26-30.

5. Liang X, Guan Y, Zhang B, et al. Severe Immune-Related Pneumonitis With PD-1 Inhibitor After Progression on Previous PD-L1 Inhibitor in Small Cell Lung Cancer: A Case Report and Review of the Literature. Front Oncol 2019;9:1437.

6. Aupérin A, Arriagada R, Pignon JP, et al. Prophylactic cranial irradiation for patients with small-cell lung cancer in complete remission. Prophylactic Cranial Irradiation Overview Collaborative Group. N Engl J Med 1999;341:476-84.

7. Slotman B, Faivre-Finn C, Kramer G, et al. Prophylactic 
cranial irradiation in extensive small-cell lung cancer. N Engl J Med 2007;357:664-72.

8. Kalemkerian GP, Loo BW, Akerley W, et al. NCCN Guidelines Insights: Small Cell Lung Cancer, Version 2.2018. J Natl Compr Canc Netw 2018;16:1171-82.

9. Takahashi T, Yamanaka T, Seto T, et al. Prophylactic cranial irradiation versus observation in patients with extensive-disease small-cell lung cancer: a multicentre, randomised, open-label, phase 3 trial. Lancet Oncol 2017;18:663-71.

10. Pezzi TA, Fang P, Gjyshi O, et al. Rates of Overall Survival and Intracranial Control in the Magnetic Resonance Imaging Era for Patients With LimitedStage Small Cell Lung Cancer With and Without Prophylactic Cranial Irradiation. JAMA Netw Open 2020;3:e201929.

11. Mamesaya N, Wakuda K, Omae K, et al. Efficacy of prophylactic cranial irradiation in patients with limiteddisease small-cell lung cancer who were confirmed to have no brain metastasis via magnetic resonance imaging after initial chemoradiotherapy. Oncotarget 2018;9:17664-74.

12. Yang $\mathrm{H}, \mathrm{Xu} \mathrm{J}$, Yao F, et al. Analysis of unexpected small cell lung cancer following surgery as the primary treatment. J Cancer Res Clin Oncol 2018;144:2441-7.

13. Yang CJ, Chan DY, Speicher PJ, et al. Surgery Versus Optimal Medical Management for N1 Small Cell Lung Cancer. Ann Thorac Surg 2017;103:1767-72.

14. Combs SE, Hancock JG, Boffa DJ, et al. Bolstering the case for lobectomy in stages I, II, and IIIA small-cell lung cancer using the National Cancer Data Base. J Thorac Oncol 2015;10:316-23.

15. Wakeam E, Acuna SA, Leighl NB, et al. Surgery Versus Chemotherapy and Radiotherapy For Early and Locally Advanced Small Cell Lung Cancer: A Propensity-Matched Analysis of Survival. Lung Cancer 2017;109:78-88.

16. $\mathrm{Xu}$ J, Yang $\mathrm{H}, \mathrm{Fu} \mathrm{X}$, et al. Prophylactic Cranial Irradiation for Patients with Surgically Resected Small Cell Lung Cancer. J Thorac Oncol 2017;12:347-53.

17. Lou Y, Zhong R, Xu J, et al. Does surgically resected small-cell lung cancer without lymph node involvement benefit from prophylactic cranial irradiation? Thorac Cancer 2020;11:1239-44.

18. Resio BJ, Hoag J, Chiu A, et al. Prophylactic cranial irradiation is associated with improved survival following resection for limited stage small cell lung cancer. J Thorac Dis 2019;11:811-8.

19. Bloom BC, Augustyn A, Sepesi B, et al. Prophylactic
Cranial Irradiation Following Surgical Resection of Early-Stage Small-Cell Lung Cancer: A Review of the Literature. Front Oncol 2017;7:228.

20. Eaton BR, Kim S, Marcus DM, et al. Effect of prophylactic cranial irradiation on survival in elderly patients with limited-stage small cell lung cancer. Cancer 2013;119:3753-60.

21. Giuliani M, Sun A, Bezjak A, et al. Utilization of prophylactic cranial irradiation in patients with limited stage small cell lung carcinoma. Cancer 2010;116:5694-9.

22. Austin PC. Statistical criteria for selecting the optimal number of untreated subjects matched to each treated subject when using many-to-one matching on the propensity score. Am J Epidemiol 2010;172:1092-7.

23. Yang CJ, Chan DY, Shah SA, et al. Long-term Survival After Surgery Compared With Concurrent Chemoradiation for Node-negative Small Cell Lung Cancer. Ann Surg 2018;268:1105-12.

24. Koh M, Song SY, Jo JH, et al. The value of prophylactic cranial irradiation in limited-stage small cell lung cancer: should it always be recommended? Radiat Oncol J 2019;37:156-65.

25. Austin PC. An Introduction to Propensity Score Methods for Reducing the Effects of Confounding in Observational Studies. Multivariate Behav Res 2011;46:399-424.

26. Elliott JA, Osterlind K, Hirsch FR, et al. Metastatic patterns in small-cell lung cancer: correlation of autopsy findings with clinical parameters in 537 patients. J Clin Oncol 1987;5:246-54.

27. Milovanovic IS, Stjepanovic M, Mitrovic D. Distribution patterns of the metastases of the lung carcinoma in relation to histological type of the primary tumor: An autopsy study Ann Thorac Med 2017;12:191-8.

28. Thomas DC, Arnold BN, Rosen JE, et al. Defining outcomes of patients with clinical stage I small cell lung cancer upstaged at surgery. Lung Cancer 2017;103:75-81.

29. Vallières E, Shepherd FA, Crowley J, et al. The IASLC Lung Cancer Staging Project: proposals regarding the relevance of TNM in the pathologic staging of small cell lung cancer in the forthcoming (seventh) edition of the TNM classification for lung cancer. J Thorac Oncol 2009;4:1049-59.

30. Sio TT, Prayongrat A, Zhang Y, et al. The Road Less Traveled: Should We Omit Prophylactic Cranial Irradiation for Patients With Small Cell Lung Cancer? Clin Lung Cancer 2018;19:289-93.

31. Le Péchoux C, Dunant A, Senan S, et al. Standard-dose versus higher-dose prophylactic cranial irradiation (PCI) 
in patients with limited-stage small-cell lung cancer in complete remission after chemotherapy and thoracic radiotherapy (PCI 99-01, EORTC 22003-08004, RTOG
0212, and IFCT 99-01): a randomised clinical trial. Lancet Oncol 2009;10:467-74.

Cite this article as: Yang $\mathrm{H}, \mathrm{Al}-\mathrm{Hurani} \mathrm{MF}, \mathrm{Xu} \mathrm{J}, \mathrm{Fan} \mathrm{L}$, Schmid RA, Zhao H, Yao F. pN1 but not pN0/N2 predicts survival benefits of prophylactic cranial irradiation in smallcell lung cancer patients after surgery. Ann Transl Med 2021;9(7):562. doi: 10.21037/atm-20-6984 
A

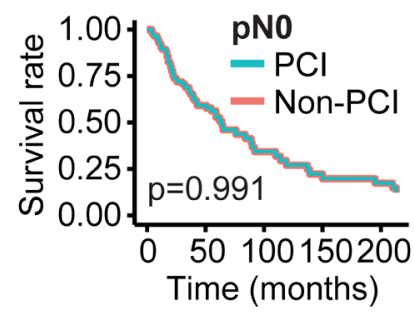

B

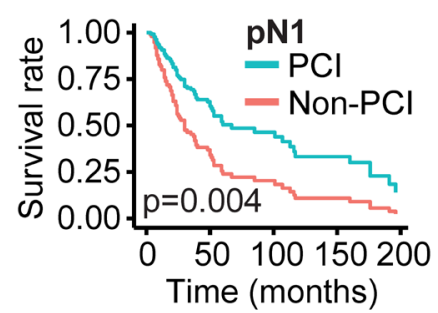

C

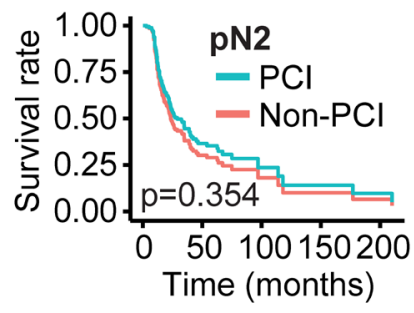

Figure S1 Survival analysis of SCLC patients with and without PCI treatment, stratified based on pN0-pN2 status after surgery. (A,B,C) Covariates-adjusted Kaplan-Meier analyses of SCLC patients with and without PCI treatment, stratified based on pN0 (A), pN1 (B), pN2 (C) after propensity matching scores (PSM).

A

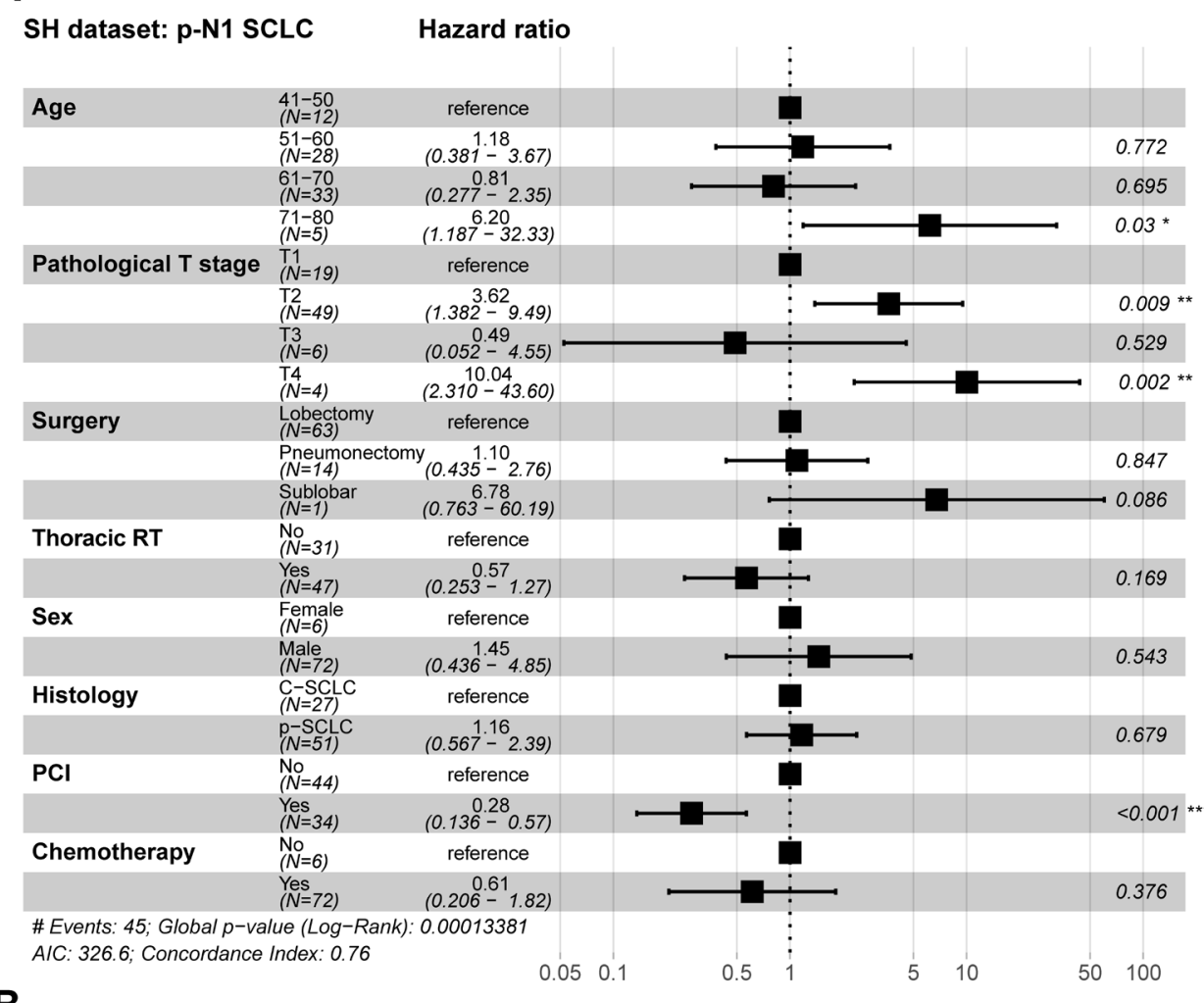

B

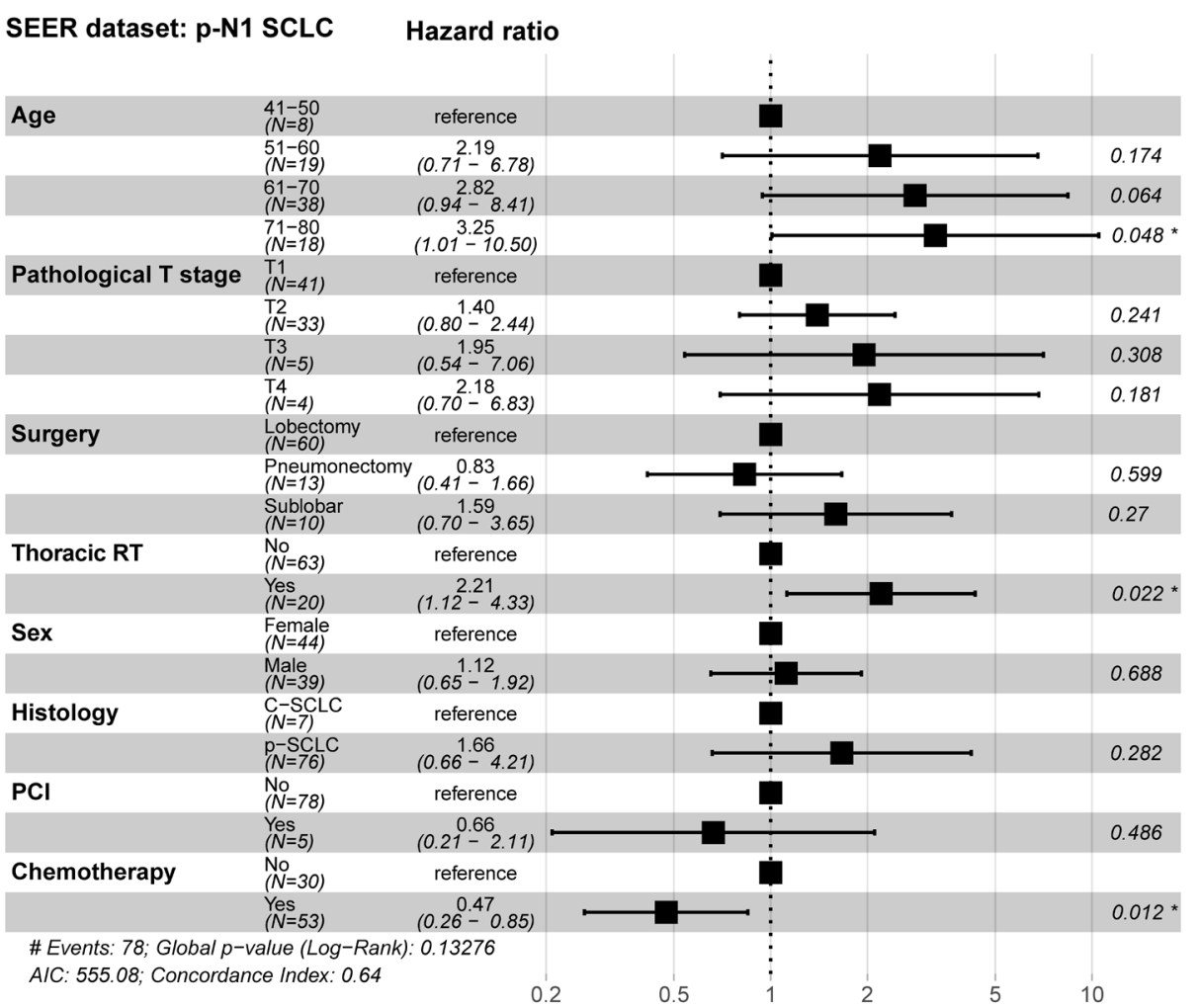

Figure S2 Separately survival analysis of SCLC patients with pN1 status after surgery. (A,B) Forest plot shows the independently prognosisinfluencing factors identified by multivariate Cox regression analysis SCLC patients with $\mathrm{pN1}$, based on single institutional (A) and SEER datasets (B), respectively. 